\title{
Organic Components of Small Bodies in the Outer Solar System: Some Results of the New Horizons Mission
}

\author{
Dale P. Cruikshank ${ }^{1, * \mathbb{D}}$, Yvonne J. Pendleton ${ }^{1}$ and William M. Grundy ${ }^{2}$ (D) \\ 1 NASA Ames Research Center, Moffett Field, CA 94035, USA; yvonne.pendleton@nasa.gov \\ 2 Lowell Observatory, Flagstaff, AZ 86001, USA; w.grundy@lowell.edu \\ * Correspondence: dale.p.cruikshank@nasa.gov
}

Received: 24 June 2020; Accepted: 24 July 2020; Published: 28 July 2020

check for updates

\begin{abstract}
The close encounters of the Pluto-Charon system and the Kuiper Belt object Arrokoth (formerly $2014 \mathrm{MU}_{69}$ ) by NASA's New Horizons spacecraft in 2015 and 2019, respectively, have given new perspectives on the most distant planetary bodies yet explored. These bodies are key indicators of the composition, chemistry, and dynamics of the outer regions of the Solar System's nascent environment. Pluto and Charon reveal characteristics of the largest Kuiper Belt objects formed in the dynamically evolving solar nebula inward of $\sim 30 \mathrm{AU}$, while the much smaller Arrokoth is a largely undisturbed relic of accretion at $\sim 45 \mathrm{AU}$. The surfaces of Pluto and Charon are covered with volatile and refractory ices and organic components, and have been shaped by geological activity. On Pluto, $\mathrm{N}_{2}, \mathrm{CO}$ and $\mathrm{CH}_{4}$ are exchanged between the atmosphere and surface as gaseous and condensed phases on diurnal, seasonal and longer timescales, while Charon's surface is primarily inert $\mathrm{H}_{2} \mathrm{O}$ ice with an ammoniated component and a polar region colored with a macromolecular organic deposit. Arrokoth is revealed as a fused binary body in a relatively benign space environment where it originated and has remained for the age of the Solar System. Its surface is a mix of $\mathrm{CH}_{3} \mathrm{OH}$ ice, a red-orange pigment of presumed complex organic material, and possibly other undetected components.
\end{abstract}

Keywords: Solar System; Pluto; Charon; Arrokoth; Kuiper Belt object; complex organics; solar nebula; protoplanetary disk; New Horizons

\section{Introduction}

The presence of complex organic molecules, often referred to as COMs, on outer Solar System planetary surfaces and in the atmosphere of Saturn's satellite Titan were first suspected from their low albedos and reddish colors. Comparisons with terrestrial kerogens and complex refractory organics synthesized by photolysis and radiolysis of gaseous mixtures of $\mathrm{CH}_{4}, \mathrm{~N}_{2}$, and other simple molecules have gradually led to the recognition that planetary processes in atmospheres and surface ices containing hydrocarbons lead to the formation of colored materials of relevance to both planetary environments and the interstellar medium, e.g., [1]. These results demonstrate that energetic processing of ices and gases containing simple organic molecules, such as $\mathrm{CH}_{4}$, readily transforms the initial carbon-bearing materials into large molecules with complex structures termed "tholin". Complex organics formed in this way often have orange and red colors that closely resemble the distinctive colors commonly found on planetary bodies [2]. This material can be sufficiently non-volatile to be considered refractory, especially for analogs that are studied in room-temperature laboratory settings, but more volatile fractions that are only stable at low temperatures likely also play a role in cold, outer Solar System settings, e.g., [3]. Earth-based and spacecraft-based spectroscopy at visible and near-infrared wavelengths have confirmed the presence of both volatile and refractory organics on several planetary 
satellites, as well as Pluto and other large Kuiper Belt objects (abbreviated herein as KBOs). Gases and organic aerosols have been confirmed in the atmosphere of Pluto by UV spectroscopy [4], while mass spectroscopy with the Cassini spacecraft has found organics in the atmosphere of Titan $[5,6]$ and the plumes of Enceladus [7]. In addition, refractory organic complexes have been found on the surfaces of Saturn's satellites Phoebe, Iapetus, and Hyperion [8], Jupiter's Ganymede and Callisto [9], Pluto and Charon [10,11], and the Kuiper Belt object (486958) $2014 \mathrm{MU}_{69}$, now named Arrokoth [12,13]. Spectra and textures of surfaces consisting of ices, silicates, and tholins have been studied in the laboratory [14].

Here we present the current understanding of the organic constituents of these bodies with emphasis on KBOs, and in particular the results of the New Horizons investigations of the Pluto-Charon system and Arrokoth, and discuss the significance of the discoveries in terms of the origins of organics in the solar nebula and the protoplanetary disk.

The relevance of Pluto, Arrokoth, and other small bodies in the outer Solar System to investigations of the origin and evolution of the early Solar System is closely related to the distinct environments in which they formed in the protoplanetary nebula. These diverse origins are reflected today in the complex dynamical structure of the Kuiper belt [15]. Present day KBOs, the remnants of a much larger early planetesimal population, occupy a disk extending from the orbit of Neptune ( $30 \mathrm{AU})$ to heliocentric distances of $\sim 50 \mathrm{AU}$ (astronomical units) and beyond. They are subdivided into classical, resonant, and scattered bodies. The classical KBOs are on relatively low inclination, low eccentricity orbits between 40 and 47 AU [16]. Their nearly circular orbits, and especially those of a sub-group of dynamically "cold" classical KBOs (CCKBOs), indicate that they have experienced little perturbation (i.e., low eccentricity and inclination) since they formed in the protoplanetary nebula [17-20]. Resonant objects occupy more dynamically excited orbits in mean-motion resonance with Neptune, with orbital periods that are in integer ratios with that of Neptune. Pluto is in the 2:3 mean-motion resonance and defines the subclass "plutinos". Many other mean-motion resonances are well-populated, too, including 2:1, 3:1, 5:1, 5:2, 5:3, 7:3, and 7:4 [21], with a distribution that extends into even higher order resonances. Scattered objects also have high inclinations and eccentricities and a broad distribution of semimajor axes but are not in resonance with Neptune. The dynamical excitation of the resonant and scattered populations reveals much about the early evolution of the Solar System, and especially an outward migration of Neptune that cleared away most of the original planetesimal disk exterior to where Neptune formed. Neptune's mean-motion resonances swept across the disk as Neptune migrated through it, resulting in capture of some planetesimals into the resonances [22-26]. The migration was driven by dynamical interaction between Neptune and the planetesimals, so the fact that Neptune stopped migrating at $30 \mathrm{AU}$ indicates the existence of some sort of transition from a more massive, densely populated planetesimal disk inside $30 \mathrm{AU}$ to a more depleted environment beyond that distance from the Sun [27].

Arrokoth is a cold classical KBO in a non-resonant orbit of low eccentricity (0.042) and low inclination $\left(2.45^{\circ}\right)$, with semi-major axis $44.58 \mathrm{AU}$ [28]. These characteristics show that it accreted in the solar nebula in this near-circular orbit, and remained at its present distance for the age of the Solar System [12]. Thus Arrokoth samples the nebula beyond the 30-AU break that terminated Neptune's migration, unlike Pluto, which, like other KBOs on more excited orbits, probably formed in the more densely populated region inside of $30 \mathrm{AU}$.

Another important class are the Centaur objects. These are an extension of the scattered population that have been recently $\left(\sim 10^{7}\right.$ y) perturbed into orbits that are rapidly evolving through interaction with the giant planets $[29,30]$. Centaurs have relatively short dynamical lifetimes $\left(\sim 10^{5}\right.$ to $\left.10^{8} \mathrm{y}\right)$ before being ejected from the Solar System or colliding with a planet. When perturbations to their orbits bring them closer to the Sun, the increased heating drives loss of mass through cometary activity [31]. A few have shown continuous cometary activity (e.g., comet $29 \mathrm{P} /$ Schwassmann-Wachmann 1 , which is also regarded as a Centaur) or episodic activity (e.g., 2060 Chiron). 


\section{Kuiper Belt Object Arrokoth}

The first in situ investigation of the nature and composition of a small Kuiper Belt object in its heliocentric region of origin was accomplished with the New Horizons spacecraft in 2019, when it flew by Arrokoth [12]. Dynamical calculations show that Arrokoth, with its near-circular and low inclination orbit, has not been substantially perturbed by the motion of the large planets over the age of the Solar System. The paucity of impact craters on its surface also points to a benign environment at that distance of $\sim 45 \mathrm{AU}$. As such, Arrokoth provides an unusually valuable window on the environment and composition of the Solar System beyond the 30-AU edge of the main planetesimal disk.

Arrokoth is a fused binary body with combined length of $36 \mathrm{~km}$ and equivalent diameter of $18 \mathrm{~km}$, and an estimated mean density of several hundred $\mathrm{kg} / \mathrm{m}^{3}$ [32,33]. The surface of Arrokoth has a near-uniform orange-red color, and its near-infrared spectrum, obtained with the New Horizons LEISA instrument, shows absorption bands at 2.1, 2.27 and $2.33 \mu \mathrm{m}$ identified as frozen methanol $\left(\mathrm{CH}_{3} \mathrm{OH}\right)$ (Figure 1). The presence of $\mathrm{H}_{2} \mathrm{O}$ ice on its surface is suggested by a shallow absorption band at $2.0 \mu \mathrm{m}$, but the band may be spurious, and the evidence for $\mathrm{H}_{2} \mathrm{O}$ is therefore ambiguous [13]. Water ice is expected on Arrokoth and all other KBOs, and has been detected spectroscopically on some, but not all, of the objects for which appropriate data exist. However, such data only exist for larger, brighter members of the more excited populations, not small cold classical KBOs like Arrokoth [34,35]. Both the steep slope of the reflectance spectrum of Arrokoth and the $\mathrm{CH}_{3} \mathrm{OH}$ bands are closely similar to those characteristics of Centaur 5145 Pholus, but Pholus also shows prominent $\mathrm{H}_{2} \mathrm{O}$ bands [36]. Another $\mathrm{KBO}, 2002 \mathrm{VE}_{95}$, a resonant plutino, also shows prominent $\mathrm{CH}_{3} \mathrm{OH}$ and $\mathrm{H}_{2} \mathrm{O}$ absorption bands [37], suggesting that the apparent absence of $\mathrm{H}_{2} \mathrm{O}$ on the surface of Arrokoth is anomalous. Whether or not ice is present on the surface, an opaque patina of space weathering is likely on these small bodies, and if present, may mask diagnostic spectral features of $\mathrm{H}_{2} \mathrm{O}$ and other possible components.

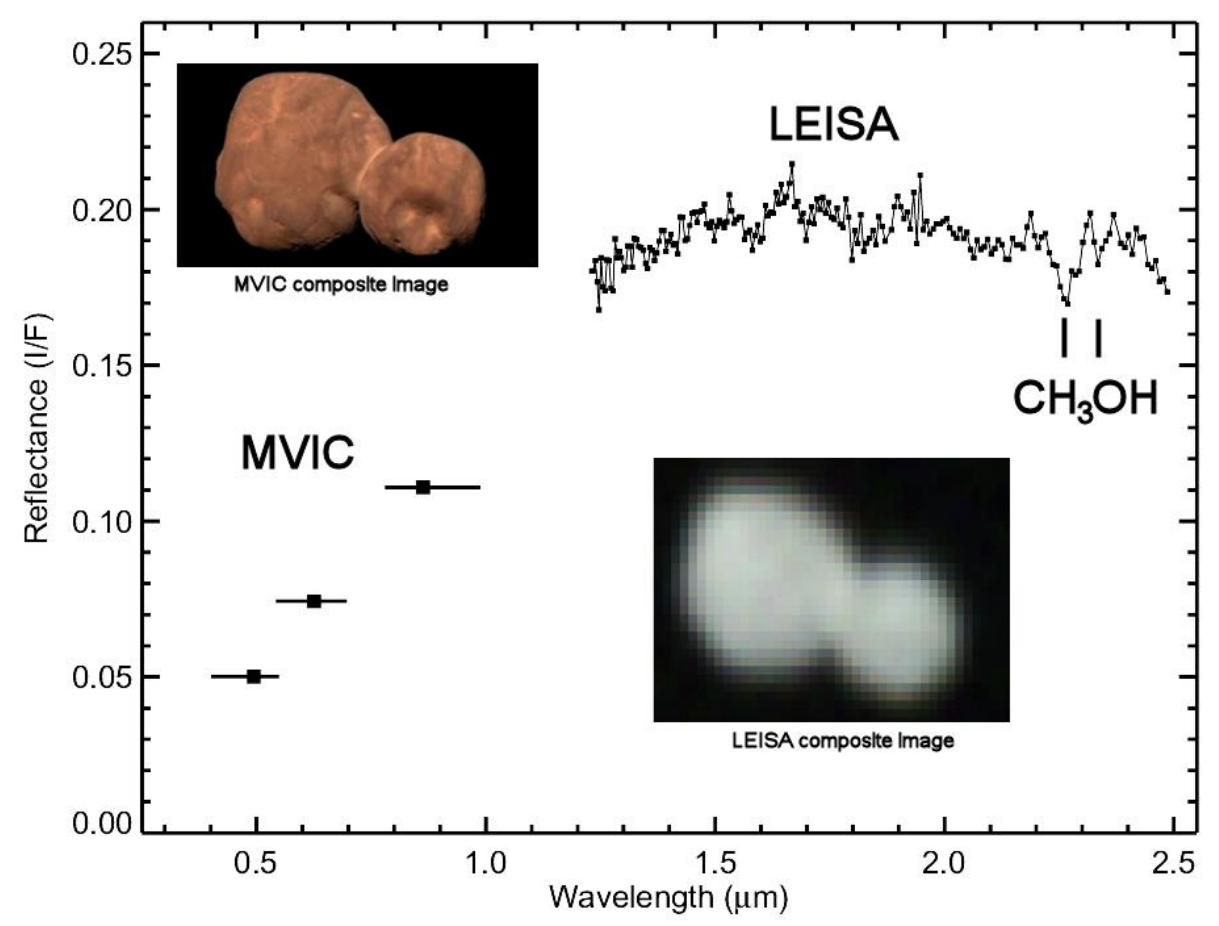

Figure 1. The spectrum and images of Arrokoth obtained with the New Horizons spacecraft. The color image was compiled from images through three color filters with the MVIC (Multispectral Visible Imaging Camera). The spectrum from 1.2 to $2.5 \mu \mathrm{m}$ was derived from spectral images with LEISA (Linear Etalon Imaging Spectral Array). The positions of the two absorption bands attributed to frozen methanol $\left(\mathrm{CH}_{3} \mathrm{OH}\right)$ are marked. The LEISA image is an rgb composite from two wavelengths, 1.6 and $2.0 \mu \mathrm{m}$. 
The dynamical stability of cold-classical KBOs over the age of the Solar System [38] demonstrates that the compositions of these bodies have not been altered by close approaches to the Sun. Furthermore, the small size of Arrokoth suggests that it has never been heated to a temperature at which it could melt and differentiate. Below the surface, the composition is expected to be a composite of simple ices, rocky chondritic material, and complex organic molecules inherited from the nascent interstellar cloud, and these may have been further processed in the protoplanetary disk $[13,33,39]$. We return to this point in Section 4.

\section{Pluto and Charon}

\subsection{Pluto}

With a radius of $1188.3 \mathrm{~km}$, Pluto is about a hundred times larger than Arrokoth and $\sim 10^{7}$ times more massive, and is representative of the largest Kuiper Belt objects. As previously described, like most other known large KBOs, also known as dwarf planets, it is likely to have accreted in the solar nebula inward of $\sim 30 \mathrm{AU}$ and was then forced to its present orbit by the outward migration of the giant planets $[23,24]$. Many of those bodies forced outward were captured into orbits resonant with Neptune, while others were scattered into orbits of high eccentricity and inclination. The formation of Pluto's large satellite Charon (radius $606 \mathrm{~km}$ ) is likely the result of a collision that left two of the bodies in locked, synchronous rotation after subsequent tidal evolution $[40,41]$ and having similar, but not identical bulk densities $\left(1854 \mathrm{~kg} / \mathrm{m}^{3}\right.$ for Pluto and $1702 \mathrm{~kg} / \mathrm{m}^{3}$ for Charon) but different surface compositions [10].

Pluto's variegated surface consists of a suite of highly volatile ices $\left(\mathrm{N}_{2}, \mathrm{CO}, \mathrm{CH}_{4}\right)$ that were originally found by near-infrared spectroscopy from ground-based telescopes [42] (Figure 2). These components were confirmed by infrared spectroscopy by the New Horizons spacecraft, and exposures of $\mathrm{H}_{2} \mathrm{O}$ ice and small amounts of frozen $\mathrm{C}_{2} \mathrm{H}_{6}$ and $\mathrm{CH}_{3} \mathrm{OH}$ were also found [10,43,44]. A non-volatile material on Pluto's surface appears to consist of complex organic molecular material deposited from the atmosphere (see below) and produced directly on the surface by photolysis and radiolysis of the surface ices that contain the hydrocarbons $\mathrm{CH}_{4}$ and $\mathrm{C}_{2} \mathrm{H}_{6}$ [44]. Pluto's variegated surface is shown in the color-enhanced image from New Horizons (Figure 3). Red-orange-colored $\mathrm{H}_{2} \mathrm{O}$ ice carries the spectral signature of $\mathrm{NH}_{3}$ hydrate, or possibly an ammoniated salt that is thought to have emerged from one or more subsurface reservoirs in episodes of cryovolcanism [45,46] (Figure 4). Beyond the surface expressions of $\mathrm{H}_{2} \mathrm{O}$ ice, there is no direct evidence of subsurface liquid water in Pluto's interior, but the case has been made by various investigators for the existence of a global ocean at some time in the planet's history, e.g., [47] on geodynamical considerations.

Grundy et al. [48] find that atmospheric aerosol tholin particles can result in thick surface deposits (several meters) over the history of Pluto. The range of colors seen on Pluto may result from chemical changes in the atmospheric tholins as they interact with the non-uniformly distributed volatiles on the planet's surface, producing distinct products in different regions [49]. Alternatively, the supply of haze particles may vary with time, and possibly location, which would also produce different products in different places. A range of materials originating from the deposition of a macromolecular organic tholin onto volatile-rich surface regions may have prebiotic chemical significance.

Pluto is a dynamic planet in terms of current geological activity, as well as the exchange of volatiles between the surface and the atmosphere. It is also chemically dynamic as photolysis and radiolysis transform simple molecules into complex, macromolecular organic materials both in the atmosphere and on the surface. The colored $\mathrm{H}_{2} \mathrm{O}$ ice that has emerged onto the surface from internal reservoirs may represent another dimension of complex organic chemistry occurring in subsurface reservoirs, where liquid water interacts with the rocky components and various organic molecules incorporated from the solar nebula during the accretion of the planet [46]. It is uncertain whether the red color represents complex organics or if it originates in water-mineral interactions. Models of the evolution of fluids in the interiors of small bodies in the outer Solar System suggest that the interaction of water 
with rock assemblages and the principal components of comets result in the formation of hydrated silicates and antifreezes (e.g., $\mathrm{NH}_{3}$ ) that prolong the liquid state as the body cools [50]. The fluid is rich in reduced $\mathrm{N}$ and $\mathrm{C}$, as well as dissolved $\mathrm{H}_{2}$, which is consistent with the presence of serpentines, ammonium phyllosilicates, and carbonates found on Ceres [51].

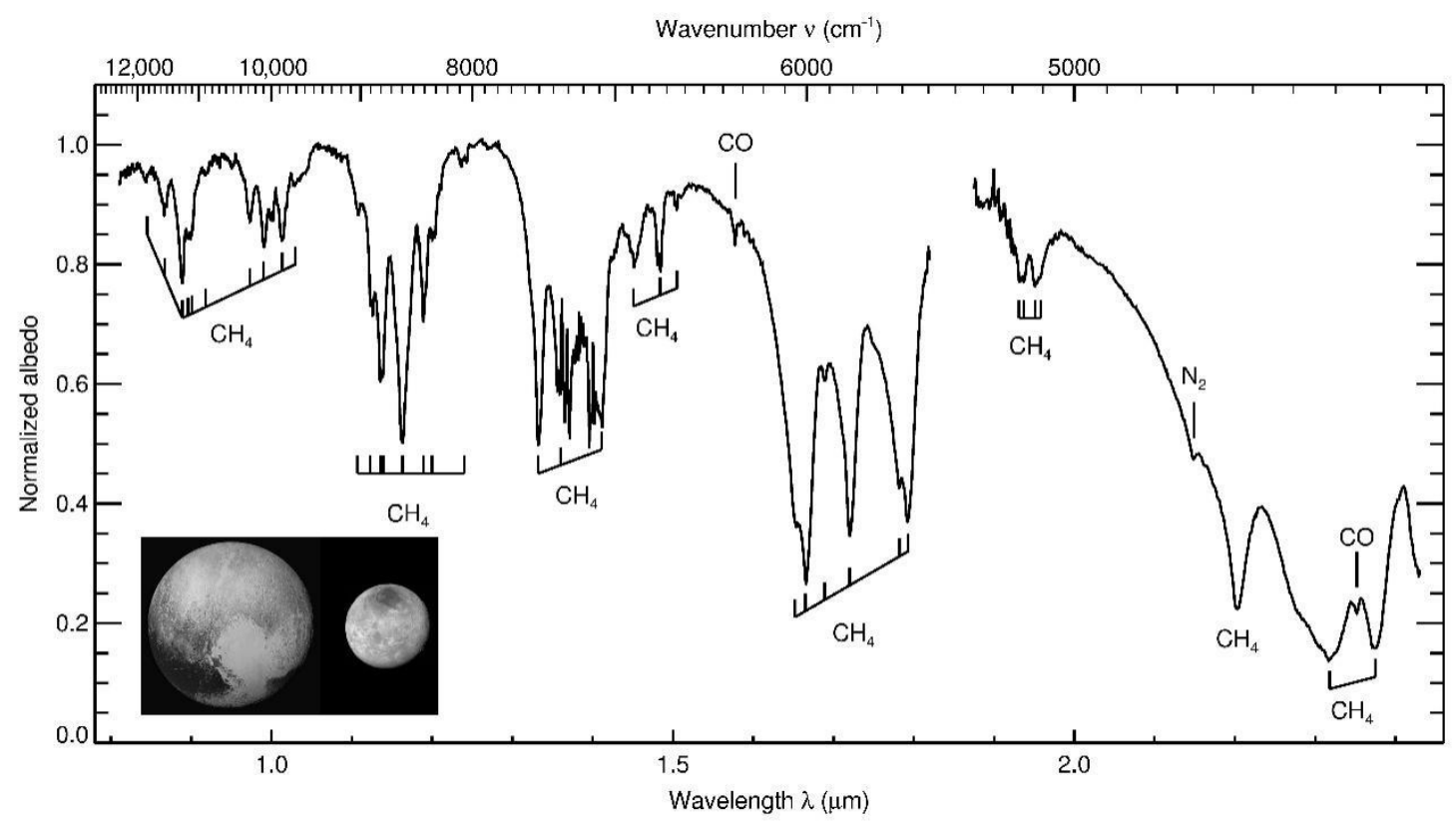

Figure 2. Infrared spectrum of Pluto (including the negligible light from Charon), shown in the inset, compiled from 20 years of ground-based telescopic observations. The identified absorption bands of $\mathrm{CH}_{4}, \mathrm{~N}_{2}$, and $\mathrm{CO}$ ices are marked. Data from B. J. Holler et al. (in preparation).

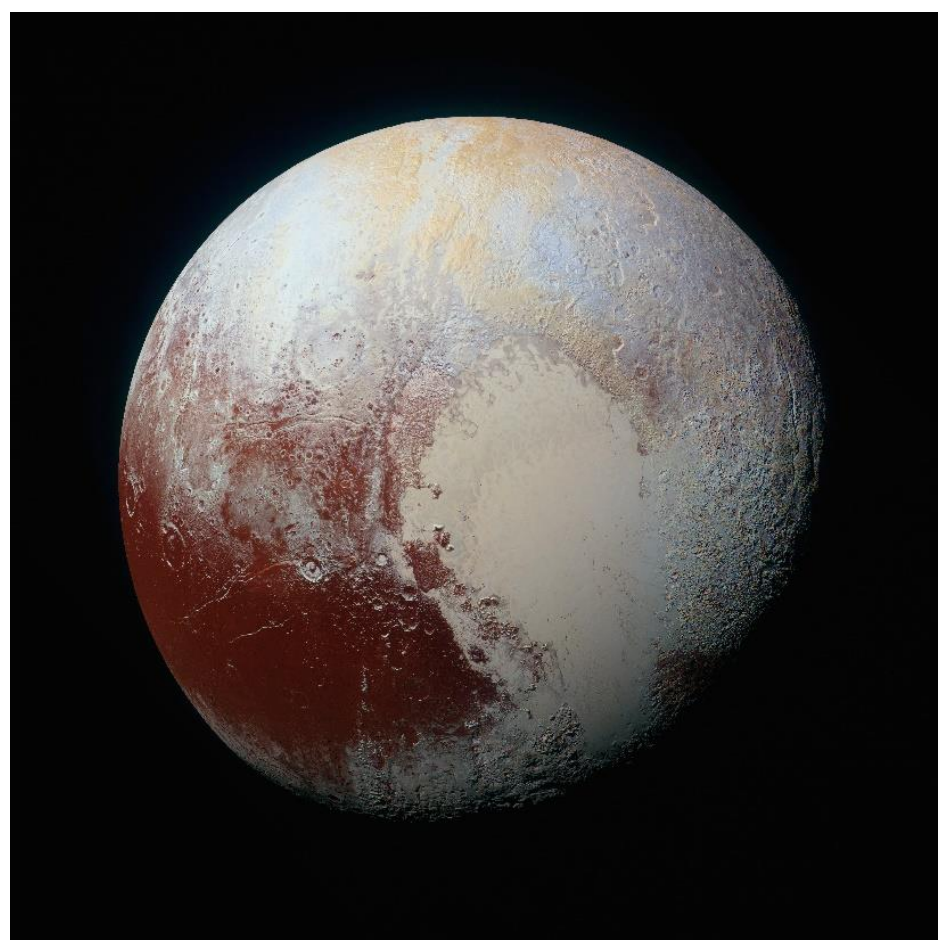

Figure 3. Color-enhanced full-disk view of the encounter hemisphere of Pluto from the New Horizons spacecraft. NASA image, courtesy of Southwest Research Institute and Johns Hopkins University. 


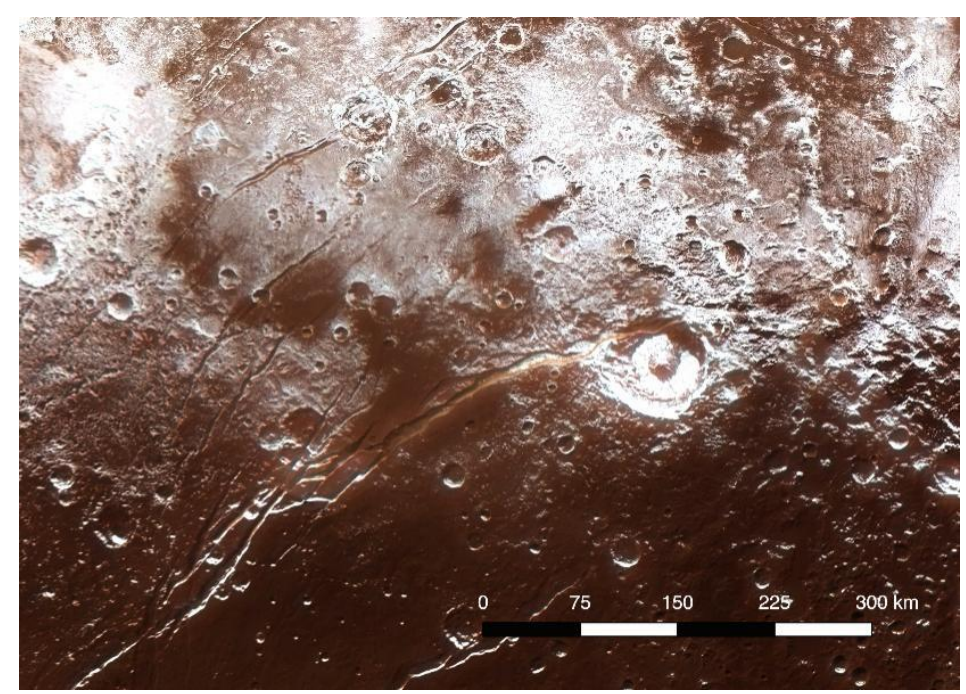

Figure 4. The region around Elliot crater (center right) and the Virgil Fossae complex, showing the strong red coloration in the main fossa trough and surrounding terrain. The red color corresponds to $\mathrm{H}_{2} \mathrm{O}$ ice that is lightly laced with an ammonia compound. NASA image, courtesy of Southwest Research Institute and Johns Hopkins University.

The formation of complex colored organic materials in fluids reacting with the organic components of chondritic meteorites [52] has been explored in the laboratory, especially in elucidating the role of formaldehyde polymer $\left(\left(\mathrm{H}_{2} \mathrm{CO}\right) \mathrm{n}\right)$ in the formation of more complex material. Formaldehyde is found in interstellar ices and was likely incorporated into the solar nebula [53]. Kebukawa et al. [54,55] showed that in experiments simulating processes in aqueous environments in planetesimals, a suite of complex amino acids can be synthesized simultaneously with insoluble organic matter. Similarly, experiments with prolonged heating of solutions of formaldehyde, glycoaldehyde, and ammonia produced a dark refractory residue. This material of high molecular weight consisted of formaldehyde polymer in addition to olefinic and aromatic molecules, and was suggested to account for the dark reddish regions on Pluto [56].

Additional experiments show that the interaction of complex organics consisting of carbon-containing moieties in chains of combinations of functional groups in a hydrous environment, particularly in the presence of $\mathrm{NH}_{3}$, produces several amino acids [57,58]. Regardless of the degree of hydration, mixtures of amino acids and other organic complexes are readily produced in a variety of environments [59]. However, while fluid environments involving $\mathrm{H}_{2} \mathrm{O}$ have existed, or possibly still exist within Pluto and other KBOs sufficiently large to have melted to some extent, very small KBOs like Arrokoth are unlikely to have ever become warm enough inside to enable hydrolysis reactions, e.g., [13,60-62].

The significance to prebiotic chemistry of Pluto's surface exposures of ammoniated $\mathrm{H}_{2} \mathrm{O}$ that contains a red pigment of presumed organic composition has been explored by Cruikshank et al. [63]. They note that laboratory experiments in which ultraviolet photolysis of water ice containing purines and pyrimidines (which have not been detected on Pluto or Arrokoth) and $\mathrm{NH}_{3}$ produces a range of nucleobases, including the five found in living systems on Earth.

\subsection{Charon and Pluto's Small Satellites}

Images and near-infrared spectra of Charon obtained during the flyby of New Horizons in $2015[10,45]$ have corroborated previous detections of $\mathrm{NH}_{3}$ or an ammoniated material on the surface found from ground-based near-infrared spectroscopy [64-66]. In addition, the spacecraft data have shown that the north polar region is tinted with an orange pigment [11] (Figure 5). The polar coloration can be explained by photolysis of $\mathrm{CH}_{4}$ deposited there during the alternating long-term darkness of each of Charon's polar regions resulting from the high obliquity of the Pluto-Charon orbit. Methane gas is escaping from Pluto's atmosphere [4], and a fraction of the streaming gas encounters Charon 
where it is cold-trapped on the polar region in darkness. That frozen $\mathrm{CH}_{4}$ is later photolyzed by solar Lyman- $\alpha$ radiation back-scattered by interplanetary hydrogen, producing a colored refractory residue (tholin). Unprocessed $\mathrm{CH}_{4}$ escapes Charon's surface as direct sunlight is slowly restored during the orbital cycle, and the process is repeated for the south polar region (which is currently in darkness) over the 248-year orbital period of Pluto and Charon around the Sun [11].

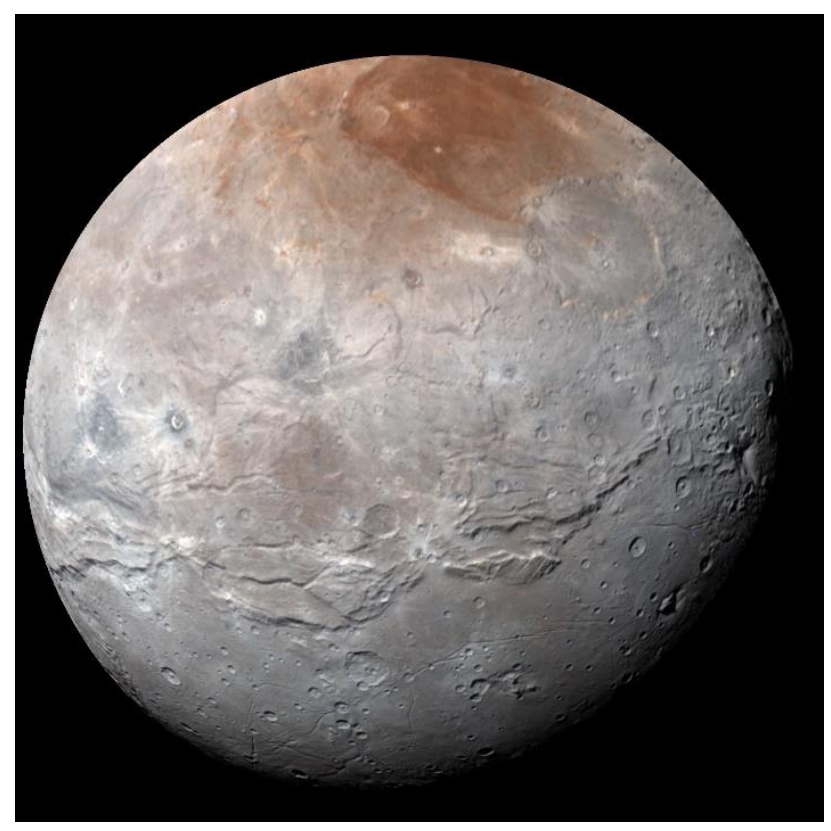

Figure 5. The Pluto-facing hemisphere of Charon, imaged with the New Horizons spacecraft, showing overall gray color with the orange tinted north polar region. NASA image, courtesy of Southwest Research Institute and Johns Hopkins University.

The orange tholin on Charon's north pole does not have diagnostic features in the region of the spectrum currently achievable from Earth-based data. In addition to ground-based spectra extending to $2.5 \mu \mathrm{m}$ obtained by Brown and Calvin [64] and other investigators, Protopapa et al. [67] recorded the spectrum to $\sim 4.05 \mu \mathrm{m}$, but at a low signal precision that did not reveal identifiable absorption bands. Although the New Horizons spacecraft obtained high-quality, spatially resolved spectral images of Charon (1.1-2.5 $\mu \mathrm{m})$, no new diagnostic bands were found [10]. Dalle Ore et al. [68] used the New Horizons data to map spatial variability in the distribution of tholin and ices across the surface, finding differences in the abundances of crystalline and amorphous $\mathrm{H}_{2} \mathrm{O}$ ice. Overall, the spectrum of Charon is well modeled by a combination of crystalline and amorphous $\mathrm{H}_{2} \mathrm{O}$ ice, an ammonia hydrate $\left(\mathrm{NH}_{3} \cdot \mathrm{H}_{2} \mathrm{O}\right)$, plus a tholin made from the electron radiolysis of a mixture of $\mathrm{CH}_{4}, \mathrm{~N}_{2}$, and CO ices [68].

In the New Horizons data, at least two of Pluto's very small satellites, Nix and Hydra, also show the presence of $\mathrm{H}_{2} \mathrm{O}$ ice, an ammonia compound that is probably a hydrate but possibly an ammonium salt, and, in the case of Nix, a distinct reddish color on one portion of the surface [69]. Whether or not the reddish color represents a deposit of complex organics is not known.

An ammonia spectral signature is also found on some exposures of $\mathrm{H}_{2} \mathrm{O}$ ice on Pluto [45,46], but as in the case of Charon and the small satellites, the identity of the ammoniated species is ambiguous. Pure ammonia ice on an exposed surface is not stable against UV irradiation, and although the stability of an ammonia hydrate is greater, it too is expected to disappear on relatively short timescales. On the basis of laboratory experiments with proton-irradiated $\mathrm{NH}_{3} \cdot \mathrm{H}_{2} \mathrm{O}$, Loeffler et al. [70] estimate that over the age of the Solar System, about $40 \%$ or more of any original ammonia on Charon has been removed from the optical surface. The stability of ammoniated salts is expected to be greater, but quantitative estimates await appropriate laboratory experiments. 
Another obstacle to a complete understanding of the surface chemistries of Charon and the small satellites is that tholins are expected to lose their color as the complex molecules are broken down by prolonged photolysis or radiolysis, eventually being converted to carbon, either in an amorphous state or as graphite. A limited laboratory study of the effect of long-term irradiation of a methane clathrate showed that as the accumulated dose of charged particles increased, the originally high-albedo colorless solid developed a distinct red color, which was eventually quenched as the albedo dropped to $\sim 10$ to $20 \%$ of its original value [71]. The overall neutral gray color of most of Charon's surface (apart from the reddish north polar region) may be explained by the long-term exposure of a veneer of organics on its surface. This situation does not apply to Pluto, where the surface is continually altered by the deposition of atmospheric aerosols in addition to the exchange of volatiles between the atmosphere and surface on seasonal and long-term timescales. The apparent indefinite retention of a red-colored region on Nix is not explained, but may hint at more recent delivery via an impact.

\section{The Formation of Arrokoth in the Solar Nebula and Contrasts to Pluto}

The detection of frozen methanol and a nearly uniform red-orange coloration across both lobes of the small body of Arrokoth are clues to its origin and evolution. The relatively benign environment in which Arrokoth formed is indicated not only by its low inclination and nearly circular orbit at $\sim 45 \mathrm{AU}$, but also by the paucity of large craters on both lobes of the body. Dynamical arguments and population studies of the cold-classical KBOs provide a convincing case for the separate formation of both lobes prior to their joining in a very gentle collision $(\sim 3 \mathrm{~m} / \mathrm{s})$ that occurred early in the formation of the Solar System [33]. The details of accretion are therefore likely to be well preserved within Arrokoth, reflected in the stability of ices found at the present time [72]. Pluto, having formed from material significantly closer to the Sun, was later perturbed outward to its current location by the migration of Neptune [23,24], providing an important contrast to the formation of Arrokoth. Pluto's much larger size (factor of about one hundred in linear dimensions and two million in volume) and tenuous atmosphere make its present-day composition decidedly different from that of Arrokoth, but comparing them can provide a context in which to place the compositions of other Kuiper Belt Objects with more complexity than Arrokoth and possibly less than Pluto.

Although $\mathrm{CH}_{3} \mathrm{OH}$ is the only ice component detected on the present-day surface of Arrokoth, its presence in the interstellar medium (ISM), e.g., [73] and in comets, e.g., [74] invites a deeper comparison. We note that $\mathrm{CH}_{3} \mathrm{OH}$ is not highly volatile and is more like $\mathrm{H}_{2} \mathrm{O}$ ice in its stability on planetary bodies. In the absence of any other plausible cause, and in view of numerous synthesis studies of tholins in the laboratory, the red-orange coloration of the surface is regarded as strongly suggestive of complex macromolecular organics found or inferred on several outer Solar System bodies [2]. Other volatile ices may exist on the surface of Arrokoth but at concentrations below the detection threshold of the LEISA spectrometer on the New Horizons spacecraft. Candidates are $\mathrm{N}_{2}$, $\mathrm{CO}$, and $\mathrm{CH}_{4}$. While these volatiles could be transiently stable on Arrokoth's coldest winter surface regions, none can survive summer surface temperatures, which range from 40 to $60 \mathrm{~K}$, nor could they survive in Arrokoth's interior, where the mean temperature is $~ 40 \mathrm{~K}$ [13]. However, some could persist trapped in clathrate form or within amorphous ice. If these species were originally present in the interior, they would have been lost from the comet's outer layers through outgassing that may have produced what appear to be collapse pits on the body's surface [13]. Pits and sinkholes are also found on comet 67P/Churumov-Gerasimenko [75,76].

The color of Arrokoth is nearly uniform, with the major exception being a lighter tone in the neck where the two bodies are fused. On many Solar System bodies, a colored surface is thought to represent a relatively thin veneer of space-weathered material. While there are no fresh craters or fault surfaces that reveal the subsurface, it is plausible that the color runs deeper than the optical surface. Considerations of surface erosion from images with resolution up to $\sim 33 \mathrm{~m} /$ pixel suggest that, over the age of Arrokoth, as much as a few meters of the surface have been removed in an ongoing process by impacts of micrometeorites from sources in the Solar System and interstellar space [77,78]. 
If this process removes material faster than photolysis and radiolysis can produce the color from native material, the color may be representative of the bulk composition of both components of Arrokoth [13]. Or, if the timescale for photolysis of native hydrocarbons is comparable to the erosion and exposure timescale, the current color of the surface represents a snapshot in time of long-term and ongoing processes (any present-day feed stocks would have to be hydrocarbons that are less volatile than methane or else be trapped in clathrate or amorphous ice, since methane ice itself is too volatile, e.g., $[13,72,79])$. If, in fact, Arrokoth accreted in the solar nebula before the disk had cleared sufficiently to expose its native feedstock to UV photolysis by the Sun, it would seem that the original material in the protoplanetary disk had already been processed sufficiently to produce the complex refractory tholins that represent the red-orange material.

\section{Forming Planetary Systems}

The proto-planetary disk (PPD) phase, which occurs prior to the development of solar systems, can be effectively modeled through a combination of the expected physical and chemical processes plus constraints offered from observational data, e.g., [80]. Dynamical movement of the gas and dust, the physical conditions of the solar nebula, and the composition of the PPD drive the chemical evolution at every stage. Chemical reactions occur as the ice-mantled dust grains cycle vertically and radially through the PPD, e.g., [81]. Observations that can spatially resolve details in some of the nearby forming systems are critical to provide insight at different stages along the way, e.g., [82].

For instance, recent observations have revealed a depletion of gas phase $\mathrm{CO}$ in the warm $(\mathrm{T}>22 \mathrm{~K})$ upper regions of young PPDs up to two orders of magnitude below the expected canonical value of $10^{-4}$, e.g., [ $[83,84]$, meaning that the solid state $\mathrm{CO}$ on the dust grains did not go back into the gas phase as thoroughly as expected. To explain the absence of the $\mathrm{CO}$ in the gas phase, investigators have suggested more $\mathrm{CO}$ than previously thought could have been sequestered on icy grains shielded in the mid-plane of the disk, e.g., [85] or that the CO may participate more fully in additional solid state chemistry that occurs on the grains through the addition of hydrogen to form products such as $\mathrm{HCO}$, $\mathrm{H}_{2} \mathrm{CO}$, methanol, etc. $[86,87]$. Indeed, the PPD models that use $\mathrm{CO}$ to form other molecules can produce methanol on the relevant timescales ( $\sim \mathrm{Myr}$ ) required if the lobes of Arrokoth incorporated methanol from the planetesimal stage. However, much work is yet to be done to understand the distribution over time and distance within the PPD that will result when the strictly physical sequestration approach is combined with the chemical evolution of $\mathrm{CO}$. Arrokoth presents an intriguing data point because it formed where we see it today, both components are thought to have fully formed as planetesimals early on, and it has existed in a benign environment over the age of the Solar System. It will be interesting to see if the future PPD models yield results that can explain the New Horizons observations of methanol on Arrokoth, not only on the required timescale, but also at the distance where it exists from our Sun. Similarly, it will be interesting to see what the future models predict for water and volatiles that were not detected (on the surface) of Arrokoth. The coupling of these sophisticated modeling efforts with future observations of distant star and planetary systems in formation, plus forthcoming new data from primitive objects in our own Solar System, will greatly advance our understanding of the origin and evolution of ices and organics in planetary systems.

Author Contributions: Each author contributed approximately equally to the research represented in this paper. Each author contributed sections to the text and figures according to their expertise. All authors have read and agreed to the published version of the manuscript.

Funding: This research was funded through NASA's New Horizons mission (DPC and WMG), and by NASA's Planetary Science Division (YJP).

Acknowledgments: This work is supported in part by NASA's New Horizons mission. The New Horizons mission owes its extraordinary success to the knowledge, skills, and hard work of the engineers, scientists, mission support specialists, and managers over the many years from its genesis to the execution of its two main goals reviewed briefly in this paper.

Conflicts of Interest: The authors declare no conflict of interest. 


\section{References}

1. Dalle Ore, C.M.; Fulchignoni, M.; Cruikshank, D.P.; Barucci, M.A.; Brunetto, R.; Campins, H.; de Bergh, C.; Debes, J.H.; Dotto, E.; Emery, J.P.; et al. Organic materials in planetary and protoplanetary systems: Nature or nurture? Astron. Astrophys. 2011, 533, A98. [CrossRef]

2. Cruikshank, D.P.; Imanaka, H.; Dalle Ore, C.M. Tholins as coloring agents on outer Solar System bodies. Adv. Space Res. 2005, 36, 178-183. [CrossRef]

3. Sakakibara, N.; Yu, P.Y.; Ito, T.; Terashima, K. Cryogenic-specific reddish coloration by cryoplasma: New explanation for color diversity of outer solar system objects. Astrophys. J. Lett. 2020, 891, L44. [CrossRef]

4. Gladstone, G.R.; Stern, S.A.; Ennico, K.; Olkin, C.B.; Weaver, H.A.; Young, L.A.; Summers, M.E.; Strobel, D.F.; Hinson, D.P.; Kammer, J.A.; et al. The atmosphere of Pluto as observed by New Horizons. Science 2016, 351, aad8866. [CrossRef]

5. Waite, J.H., Jr.; Young, D.T.; Cravens, T.E.; Coates, A.J.; Crary, F.J.; Magee, B.; Westlake, J. The process of tholin formation in Titan's upper atmosphere. Science 2007, 316, 870-875. [CrossRef]

6. Brassé, C.; Muñoz, O.; Coll, P.; Rauln, F. Optical constants of Titan aerosols and there tholins analogs: Experimental results and modeling/observational data. Planet. Space Sci. 2015, 109, 159-174. [CrossRef]

7. Postberg, F.; Khawaja, N.; Abel, B.; Choblet, G.; Glein, C.R.; Gudipati, M.S.; Henderson, B.L.; Hsu, H.W.; Kempf, S.; Klenner, F.; et al. Macromolecular organic compounds from the depths of Enceladus. Nature 2018, 588, 564-568. [CrossRef]

8. Cruikshank, D.P.; Wegryn, E.; Dalle Ore, C.M. Hydrocarbons on Saturn's satellites Iapetus and Phoebe. Icarus 2008, 193, 334-343. [CrossRef]

9. McCord, T.B.; Carlson, R.W.; Smythe, W.D.; Hansen, G.D.; Clark, R.N.; Hibbitts, C.A.; Fanale, F.P.; Granahan, J.C.; Segura, M.; Matson, D.L.; et al. Organics and other molecules in the surfaces of Callisto and Ganymede. Science 1997, 278, 271-275. [CrossRef]

10. Grundy, W.M.; Binzel, R.P.; Buratti, B.J.; Cook, J.C.; Cruikshank, D.P.; Dalle Ore, C.M.; Earle, A.M.; Ennico, K.; Howett, C.J.A.; Lunsford, A.W.; et al. Surface compositions across Pluto and Charon. Science 2016, 351, aad9189. [CrossRef]

11. Grundy, W.M.; Cruikshank, D.P.; Gladstone, G.R.; Howett, C.J.A.; Lauer, T.R.; Spencer, J.R.; Summers, M.E.; Buie, M.W.; Earle, A.M.; Ennico, K.; et al. Formation of Charon's red polar caps. Nature 2016, 539, 65-68. [CrossRef] [PubMed]

12. Stern, S.A.; Weaver, H.A.; Spencer, J.R.; Olkin, C.B.; Gladstone, G.R.; Grundy, W.M.; Moore, J.M.; Cruikshank, D.P.; Elliott, H.A.; McKinnon, W.B.; et al. Initial results from the New Horizons exploration of $2014 \mathrm{MU}_{69}$, a small Kuiper Belt object. Science 2019, 364, eaaw9771. [CrossRef] [PubMed]

13. Grundy, W.M.; Bird, M.K.; Britt, D.T.; Coo, J.C.; Cruikshank, D.P.; Howett, C.J.A.; Krijt, S.; Linscott, I.R.; Olkin, C.B.; Parker, A.H.; et al. Color, composition, and thermal environment of Kuiper Belt object (486958) Arrokoth. Science 2020, 367, eaay3705. [CrossRef] [PubMed]

14. Poch, O.; Pommerol, A.; Jost, B.; Carrasco, N.; Szopa, C.; Thomas, N. Sublimation of water ice mixed with silicates and tholins: Evolution of surface texture and reflectance spectra, with implications for comets. Icarus 2016, 267, 154-173. [CrossRef]

15. Gladman, B.; Marsden, B.G.; VanLaerhoven, C. Nomenclature in the outer Solar System. In The Solar System Beyond Neptune; Barucci, M.A., Ed.; University of Arizona Press: Tucson, AZ, USA, 2008; pp. 43-57.

16. Petit, J.M.; Kavelaars, J.J.; Gladman, B.J.; Jones, R.L.; Parker, J.W.; van Laerhoven, C.; Nicholson, P.; Mars, G.; Rousselot, P.; Mousis, O.; et al. The Canada-France Ecliptic Plane Survey-Full data release: The orbital structure of the Kuiper belt. Astron. J. 2011, 142, 131. [CrossRef]

17. Batygin, K.; Brown, M.E.; Fraser, W.C. Retention of a primordial cold classical Kuiper belt in an instability-driven model of Solar System formation. Astrophys. J. 2011, 738, 13. [CrossRef]

18. Dawson, R.I.; Murray-Clay, R. Neptune's wild days: Constraints from the eccentricity distribution of the classical Kuiper belt. Astrophys. J. 2012, 750, 43. [CrossRef]

19. Wolff, S.; Dawson, R.; Murray-Clay, R.A. Neptune on tiptoes: Dynamical histories that preserve the cold classical Kuiper belt. Astrophys. J. 2012, 746, 171. [CrossRef]

20. de Sousa, R.R.; Gomes, R.; Morbidelli, A.; Vieira Neto, E. Dynamical effects on the classical Kuiper belt during the excited-Neptune model. Icarus 2019, 334, 89-98. [CrossRef] 
21. Gladman, B.; Lawler, S.M.; Petit, J.M.; Kavelaars, J.; Jones, R.L.; Parker, J.W.; van Laerhoven, C.; Nicholson, P.; Rousselot, P.; Bieryla, A.; et al. The resonant transneptunian populations. Astron. J. 2012, 144, 23. [CrossRef]

22. Malhotra, R. The origin of Pluto's orbit: Implications for the solar system beyond Neptune. Astron. J. 1995, 110, 420-429. [CrossRef]

23. Nesvorný, D. Jumping Neptune can explain the Kuiper belt kernel. Astron. J. 2015, 150, 68. [CrossRef]

24. Nesvorný, D. Evidence for slow migration of Neptune from the inclination of Kuiper Belt objects. Astron. J. 2015, 150, 73. [CrossRef]

25. Kaib, N.A.; Sheppard, S.S. Tracking Neptune's migration history through high-perihelion resonant transneptunian objects. Astron. J. 2016, 152, 133. [CrossRef]

26. Lawler, S.M.; Pike, R.E.; Kaib, N.; Alexandersen, M.; Bannister, M.T.; Chen, Y.T.; Gladman, B.; Gwyn, S.; Kavelaars, J.J.; Petit, J.M.; et al. OSSOS XIII: Fossilized resonant dropouts tentatively confirm Neptune's migration was grainy and slow. Astron. J. 2019, 153, 253. [CrossRef]

27. Shannon, A.; Wu, Y.; Lithwick, Y. Forming the cold classical Kuiper belt in a light disk. Astrophys. J. 2016, 818, 175. [CrossRef]

28. Porter, S.B.; Buie, M.W.; Parker, A.H.; Spencer, J.R.; Benecchi, S.; Tanga, P.; Verbiscer, A.; Kavelaars, J.J.; Gwyn, S.D.J.; Young, E.F.; et al. High-precision orbit fitting and uncertainty analysis of (486958) $2014 \mathrm{MU}_{69}$. Astron. J. 2018, 156, 20. [CrossRef]

29. Tiscareno, M.S.; Malhotra, R. The dynamics of known Centaurs. Astron. J. 2003, 126, 3122-3131. [CrossRef]

30. Volk, K.; Malhotra, R. Do Centaurs preserve their source inclinations? Icarus 2013, 224, 66-73. [CrossRef]

31. Jewitt, D. The active Centaurs. Astron. J. 2009, 137, 4296-4312. [CrossRef]

32. Spencer, J.R.; Stern, S.A. The geology and geophysics of Kuiper Belt object (486948) Arrokoth. Science 2020, 367, eaay3999. [CrossRef] [PubMed]

33. McKinnon, W.B.; Richardson, D.C.; Marohnic, J.C.; Keane, J.T.; Grundy, W.M.; Hamilton, D.P.; Nesvorný, D.; Umurhan, O.M.; Lauer, T.R.; Singer, K.N.; et al. The solar nebula origin of (486958) Arrokoth, a primordial contact binary in the Kuiper Belt. Science 2020, 367, eaay6620. [CrossRef] [PubMed]

34. Barucci, M.A.; Brown, M.E.; Emery, J.P.; Merlin, F. Composition and surface properties of transneptunian objects and centaurs. In The Solar System Beyond Neptune; Barucci, M.A., Ed.; University of Arizona Press: Tucson, AZ, USA, 2008; pp. 143-160.

35. Barucci, M.A.; Merlin, F. Surface composition of trans-neptunian objects. In The Trans-Neptunian Solar System; Prialnik, D., Barucci, M.A., Young, L.A., Eds.; Elsevier: Amsterdam, The Netherlands, 2020; 464p.

36. Cruikshank, D.P.; Roush, T.L.; Bartholomew, M.J.; Geballe, T.R.; Pendleton, Y.J.; White, S.M.; Bell, J.F., III; Davies, J.K.; Owen, T.C.; de Bergh, C.; et al. The composition of centaur 5145 Pholus. Icarus 1998, 135, 389-407. [CrossRef]

37. Barucci, M.A.; Perna, D.; Alvarez-Candal, A.; Müller, T.; Mommert, M.; Kiss, C.; Fornasier, S.; Santos-Sanz, P.; Dotto, E. The extra red plutino (55638) $2002 \mathrm{VE}_{95}$. Astron. Astrophys. 2012, 539, A152. [CrossRef]

38. Batygin, K.; Brown, M.E.; Betts, H. Instability-driven dynamical evolution model of a five-planet outer solar system. Astrophys. J. Lett. 2012, 744, L3. [CrossRef]

39. Pendleton, Y.J.; Cruikshank, D.P.; Stern, S.A.; Dalle Ore, C.M.; Grundy, W.; Materese, C.; Protopapa, S.; Schmitt, B.; Lisse, C.L. Kuiper Belt object 2014MU69, Pluto, and Phoebe as windows on the composition of the early solar nebula. In Laboratory Astrophysics: In Observations to Interpretation; Proc. IAU Symp. 350; Salama, F., Linnartz, H., Eds.; 2020; in press. [CrossRef]

40. Canup, R.M. On a giant impact origin of Charon, Nix, and Hydra. Astron. J. 2011, 141, 35-44. [CrossRef]

41. McKinnon, W.B.; Stern, S.A.; Weaver, H.A.; Nimmo, F.; Bierson, C.J.; Grundy, W.M.; Cook, J.C.; Cruikshank, D.P.; Parker, A.H.; Moore, J.M.; et al. Origin of the Pluto-Charon system: Constraints from the New Horizons flyby. Icarus 2017, 287, 2-11. [CrossRef]

42. Cruikshank, D.P.; Grundy, W.M.; DeMeo, F.E.; Buie, M.W.; Binzel, R.P.; Jennings, D.E.; Olkin, C.B.; Parker, J.W.; Reuter, D.C.; Spencer, J.R.; et al. The surface compositions of Pluto and Charon. Icarus 2015, 246, 82-92. [CrossRef]

43. Stern, S.A.; Bagenal, F.; Ennico, K.; Gladstone, G.R.; Grundy, W.M.; McKinnon, W.B.; Moore, J.M.; Olkin, C.B.; Spencer, J.R.; Weaver, H.A.; et al. The Pluto system: Initial results from its exploration by New Horizons. Science 2015, 350, 1815. [CrossRef] 
44. Cook, J.C.; Dalle Ore, C.M.; Protopapa, S.; Binzel, R.P.; Cruikshank, D.P.; Earle, A.; Grundy, W.M.; Ennico, K.; Howett, C.; Jennings, D.E.; et al. The distribution of $\mathrm{H}_{2} \mathrm{O}, \mathrm{CH}_{3} \mathrm{OH}$, and hydrocarbon-ices on Pluto: Analysis of New Horizons spectral images. Icarus 2019, 331, 148-169. [CrossRef]

45. Dalle Ore, C.M.; Cruikshank, D.P.; Protopapa, S.; Scipioni, F.; McKinnon, W.B.; Cook, J.C.; Grundy, W.M.; Schmitt, B.; Stern, S.A.; Moore, J.M.; et al. Detection of ammonia on Pluto's surface in a region of geologically recent tectonism. Sci. Adv. 2019, 5. [CrossRef] [PubMed]

46. Cruikshank, D.P.; Umurhan, O.M.; Beyer, R.A.; Schmitt, B.; Keane, J.T.; Runyon, K.D.; Atri, D.; White, O.L.; Matsuyama, I.; Moore, J.M.; et al. Recent cryovolcanism in Virgil Fossae on Pluto. Icarus 2019, 330, 155-168. [CrossRef]

47. Nimmo, F.; Hamilton, D.P.; McKinnon, W.B.; Schenk, P.M.; Binzel, R.P.; Bierson, C.J.; Beyer, R.A.; Moore, J.M.; Stern, S.A.; Weaver, H.A.; et al. Reorientation of Sputnik Planitia implies a subsurface ocean on Pluto. Nature 2016, 540, 94-96. [CrossRef] [PubMed]

48. Grundy, W.M.; Bertrand, T.; Binzel, R.P.; Buie, M.W.; Buratti, B.J.; Cheng, A.F.; Cook, J.C.; Cruikshank, D.P.; Devins, S.L.; Ore, C.M.D.; et al. Pluto's haze as a surface material. Icarus 2018, 314, 232-245. [CrossRef]

49. Protopapa, S.; Olkin, C.B.; Grundy, W.M.; Li, J.Y.; Verbiscer, A.; Cruikshank, D.P.; Gautier, T.; Quirico, E.; Cook, J.C.; Reuter, D.; et al. Disk-resolved photometric properties of Pluto and the coloring materials across its surface. Astron. J. 2020, 159, 74. [CrossRef]

50. Neveu, M.; Desch, S.J.; Castillo-Rogez, J.C. Aqueous geochemistry in icy world interiors: Equilibrium fluid, rock, and gas compositions, and fate of antifreezes and radionuclides. Geochim. Cosmochim. Acta 2017, 212, 324-371. [CrossRef]

51. De Sanctis, M.C.; Ammannito, E.; Raponi, A.; Marchi, S.; McCord, T.B.; McSween, H.Y.; Capaccioni, F.; Capria, M.T.; Carrozzo, F.G.; Ciarniello, M.; et al. Ammoniated phyllosilicates with a likely outer solar system origin on (1) Ceres. Nature 2015, 528, 241. [CrossRef]

52. Alexander, C.M.; Russell, S.S.; Arden, J.W.; Ash, R.D.; Grady, M.M.; Pillinger, C.T. The origin of chondritic macromolecular organic matter: A carbon and nitrogen isotope study. Meteorit. Planet. Sci. 1998, 33, 603-622. [CrossRef]

53. Schutte, W.A.; Gerakines, P.A.; Geballe, T.R.; van Dishoeck, E.F.; Greenberg, J.M. Discovery of solid formaldehyde toward the protostar GL 2136: Observations and laboratory simulation. Astron. Astrophys. 1996, 309, 633-647.

54. Kebukawa, Y.; Cody, G.D. A kinetic study of the formation of organic solids from formaldehyde: Implications for the origin of extraterrestrial organic solids in primitive Solar System objects. Icarus 2015, 248, 412-423. [CrossRef]

55. Kebukawa, Y.; Chan, Q.H.S.; Tachibana, S.; Kobayashi, K.; Zolensky, M.E. One-pot synthesis of amino acid precursors with insoluble organic matter in planetesimals with aqueous activity. Sci. Adv. 2017, 3. [CrossRef]

56. Sekine, Y.; Genda, H.; Kamata, S.; Funatsu, T. The Charon-forming giant impact as a source of Pluto's dark equatorial regions. Nat. Astron. 2017, 1, 31. [CrossRef]

57. Neish, C.D.; Somogyi, Á.; Lunine, J.I.; Smith, M.A. Low temperature hydrolysis of laboratory tholins in ammonia water solutions: Implications for prebiotic chemistry on Titan. Icarus 2009, 201, 412-421. [CrossRef]

58. Neish, C.D.; Somogyi, Á.; Smith, M.A. Titan's primordial soup: Formation of amino acids via low-temperature hydrolysis of tholins. Astrobiology 2010, 10, 337-347. [CrossRef] [PubMed]

59. Cleaves, H.J., II; Neish, C.; Callahan, M.P.; Parker, E.; Fernandez, F.M.; Dworkin, J.P. Amino acids generated from hydrated Titan tholins: Comparison with Miller-Urey electric discharge products. Icarus 2014, 237, 182-189. [CrossRef]

60. Prialnik, D.; Sarid, G.; Rosenberg, E.D.; Merk, R. Thermal and chemical evolution of comet nuclei and Kuiper belt objects. Space Sci. Rev. 2008, 138, 147-164. [CrossRef]

61. Bierson, C.J.; Nimmo, F. Using the density of Kuiper belt objects to constrain their composition and formation history. Icarus 2019, 326, 10-17. [CrossRef]

62. Grundy, W.M.; Noll, K.S.; Buie, M.W.; Benecchi, S.D.; Ragozzine, D.; Roe, H.G. The mutual orbit, mass, and density of transneptunian binary G!kúnll'hòmdímà (229762 2007 UK $_{126}$ ). Icarus 2019, 334, 30-38. [CrossRef]

63. Cruikshank, D.P.; Materese, C.K.; Pendleton, Y.J.; Boston, P.J.; Grundy, W.M.; Schmitt, B.; Lisse, C.M.; Runyon, K.D.; Keane, J.T.; Beyer, R.A.; et al. Prebiotic chemistry of Pluto. Astrobiology 2019, 17, 7. [CrossRef]

64. Brown, M.E.; Calvin, W.M. Evidence for crystalline water and ammonia ices on Pluto's satellite Charon. Science 2000, 287, 107-109. [CrossRef] 
65. Dumas, C.; Terrile, R.J.; Brown, R.H.; Schneider, G.; Smith, B.A. Hubble Space Telescope NICMOS spectroscopy of Charon's leading and trailing hemispheres. Astron. J. 2001, 121, 1163-1170. [CrossRef]

66. Cook, J.C.; Desch, S.J.; Roush, T.L.; Trujillo, C.A.; Geballe, T.R. Near-infrared spectroscopy of Charon: Possible evidence for cryovolcanism on Kuiper belt objects. Astrophys. J. 2007, 663, 1406-1419. [CrossRef]

67. Protopapa, S.; Boehnhardt, H.; Herbst, T.M.; Cruikshank, D.P.; Grundy, W.M.; Merlin, F.; Olkin, C.B. Surface characterization of Pluto and Charon by L and M band spectra. Astron. Astrophys. 2008, 490, 365-375. [CrossRef]

68. Dalle Ore, C.M.; Protopapa, S.; Cook, J.C.; Grundy, W.M.; Cruikshank, D.P.; Verbiscer, A.J.; Ennico, K.; Olkin, C.B.; Stern, S.A.; Weaver, H.A.; et al. Ices on Charon: Distribution of $\mathrm{H}_{2} \mathrm{O}$ and $\mathrm{NH}_{3}$ from New Horizons LEISA observations. Icarus 2017, 300, 21-32. [CrossRef]

69. Cook, J.C.; Dalle Ore, C.M.; Protopapa, S.; Binzel, R.P.; Cartwright, R.; Cruikshank, D.P.; Earle, A.; Grundy, W.M.; Ennico, K.; Howett, C.; et al. Composition of Pluto's small satellites: Analysis of New Horizons spectral images. Icarus 2018, 315, 30-45. [CrossRef]

70. Loeffler, M.J.; Raut, U.; Baragiola, R.A. Radiation chemistry in ammonia-water ices. J. Chem. Phys. 2010, 132, 054508. [CrossRef]

71. Thompson, W.R.; Murray, B.G.J.P.T.; Khare, B.N.; Sagan, C. Coloration and darkening of methane clathrate and other ices by charged particle irradiation. Applications to the outer Solar System. J. Geophys. Res. 1987, 92, 933-947. [CrossRef]

72. Lisse, C.M.; Young, L.A.; Cruikshank, D.P.; Sandford, S.A.; Stern, A.; Weaver, H.A., Jr.; Umurhan, O.M.; Pendleton, Y.J.; Keane, J.T.; Gladstone, R.; et al. On the origin and stability of Arrokoth's and Pluto's ices. Icarus 2020, in review.

73. Boogert, A.C.A.; Huard, T.L.; Cook, A.M.; Chiar, J.E.; Knez, C.; Decin, L.; Blake, G.A.; Tielens, A.G.G.M.; van Dishoeck, E.F. Ice and dust in the quiescent medium of isolated dense cores. Astrophys. J. 2011, 729, 92. [CrossRef]

74. Mumma, M.J.; DiSanti, M.A.; Dello Russo, N.; Magee-Sauer, K.; Gibb, E.; Novak, R. Remote infrared observations of parent volatiles in comets: A window on the early solar system. Adv. Space Res. 2003, 31, 2563-2575. [CrossRef]

75. Mousis, O.; Guilbert-Lepoutre, A.; Brugger, B.; Jorda, L.; Kargel, J.S.; Bouquet, A.; Auger, A.T.; Lamy, P.; Vernazza, P.; Thomas, N.; et al. Pits formation from volatile outgassing on 67P/Churyumov-Gerasimenko. Astrophys. J. Lett. 2015, 814. [CrossRef]

76. Vincent, J.B.; Bodewits, D.; Besse, S.; Sierks, H.; Barbieri, C.; Lamy, P.; Rodrigo, R.; Koschny, D.; Rickman, H.; Keller, H.U.; et al. Large heterogeneities in comet $67 \mathrm{P}$ as revealed by active pits from sinkhole collapse. Nature 2015, 523, 63-66. [CrossRef]

77. Stern, S.A. ISM-induced erosion and gas-dynamical drag in the Oort cloud. Icarus 1990, 84, $447-466$. [CrossRef]

78. Stern, S.A. The evolution of comets in the Oort cloud and Kuiper belt. Nature 2003, 424, 639-642. [CrossRef] [PubMed]

79. Schaller, E.L.; Brown, M.E. 2007. Volatile loss and retention on Kuiper belt objects. Astrophys. J. 2007, 659, L61-L64. [CrossRef]

80. Pontoppidan, K.M.; Salyk, C.; Bergin, E.A. Volatiles in protoplanetary disks. In Protostars and Planets VI; Beuther, H., Klessen, R.S., Cornelis, P., Henning, T., Eds.; Univ. Arizona Press: Tucson, AZ, USA, 2014; pp. 363-385.

81. Ciesla, F.J.; Sandford, S.A. Organic synthesis via irradiation and warming of ice grains in the solar nebula. Science 2012, 336, 452-454. [CrossRef] [PubMed]

82. Brogan, C.L.; Perez, L.M.; Hunter, T.R.; Dent, W.R.F.; Hales, A.S.; Hills, R.E.; Corder, S.; EFomalont, B.; Vlahakis, C.; ALMA Partnership; et al. The 2014 ALMA long baseline campaign: First results from high angular resolution observations toward the HL Tau region. Astrophys. J. Lett. 2015, 808, L3.

83. McClure, M.K.; Bergin, E.A.; Cleeves, L.I.; van Dishoeck, E.F.; Blake, G.A.; Evans, N.J., II; Green, J.D.; Henning, T.; Öberg, K.I.; Pontoppidan, K.M.; et al. Mass measurements in protoplanetary disks from hydrogen deuteride. Astrophys. J. 2016, 831, 167. [CrossRef]

84. Zhang, K.; Bergin, E.A.; Blake, G.A.; Cleeves, L.; Schwarz, K.R. Mass inventory of the giant-planet formation zone in a solar nebula analogue. Nat. Astron. 2017, 1, 130. [CrossRef] 
85. Krijt, S.; Schwarz, K.; Bergin, E.A.; Ciesla, F.J. Transport of CO in protoplanetary disks: Consequences of pebble formation, settling, and radial drift. Astrophys. J. 2018, 864, 78. [CrossRef]

86. Bergin, E.A.; Cleeves, L.; Crockett, N.; Blake, G. Exploring the origins of carbon in terrestrial worlds. Faraday Discuss. 2014, 168, 61-79. [CrossRef] [PubMed]

87. Bosman, A.D.; Walsh, C.; van Dishoeck, E.F. CO destructiion in protoplanetary disk midplanes: Inside versus outside the CO snow surface. Astron. Astrophys. 2018, 618, A182. [CrossRef]

(C) 2020 by the authors. Licensee MDPI, Basel, Switzerland. This article is an open access article distributed under the terms and conditions of the Creative Commons Attribution (CC BY) license (http://creativecommons.org/licenses/by/4.0/). 